\title{
Generadores de electricidad bioinorgánicos. Conversión de energía química renovable a través de materiales piezoeléctricos funcionalizados con enzimas
}

\author{
Susana Velasco Lozano \\ Investigadora posdoctoral del Instituto de Síntesis Química y \\ Catálisis Homogénea de la Universidad de Zaragoza-CSIC \\ svelasco@unizar.es

\section{Fernando López Gallego} \\ Investigador asociado del Instituto de Síntesis Química y \\ Catálisis Homogénea de la Universidad de Zaragoza-CSIC e investigador de la \\ Fundación Agencia Aragonesa para la Investigación y el Desarrollo (ARAID) \\ flopezgallego@unizar.es
}

\section{Extracto}

En este proyecto hemos desarrollado una nueva generación de generadores bioinorgánicos para producir electricidad a partir de combustibles renovables. Estos sistemas se presentan como alternativa a las pilas de combustible basadas en procesos electroquímicos. En este contexto, hemos fabricado un nuevo sistema híbrido que fusiona una maquinaria biológica, capaz de transformar un combustible en energía mecánica (presión y vibración), con un material piezoeléctrico que transforma esa energía mecánica en electricidad (generador bioinorgánico). Los estímulos mecánicos de presión y vibración son generados gracias a la producción de gases a partir de combustibles. Esta reacción está catalizada por la maquinaria biológica (enzimas) que forma parte del generador. Estos dispositivos son capaces de producir electricidad a escala microscópica a partir de combustibles procedentes de fuentes renovables como azúcares y alcoholes provenientes de la biomasa. Este hecho es muy significativo, puesto que indirectamente permite generar electricidad con cero emisiones. Además, estos generadores bioinorgánicos abren un abanico apasionante de nuevas aplicaciones en campos tan diversos como la sensórica, la robótica y la energía.

Palabras clave: enzimas; biocatálisis; energía; piezoelectricidad; combustibles renovables.

Cómo citar: Velasco Lozano, S. y López Gallego, F. Generadores de electricidad bioinorgánicos. Conversión de energía química renovable a través de materiales piezoeléctricos funcionalizados con enzimas. Tecnología, Ciencia y Educación, 15, 125-139. 


\title{
Bio-inorganic electricity generators. Conversion of renewable chemical energy driven by enzyme-functionalized piezoelectric materials
}

\author{
Susana Velasco Lozano \\ Fernando López Gallego
}

\begin{abstract}
In this project we have developed a new generation of bio-inorganic generators aimed at the electricity production from renewable fuels. These systems are presented as an alternative to electrochemical fueled cells. In this context, we have fabricated a new hybrid system, which fuses a biological machinery able to transform chemical energy into mechanical energy (pressure and vibration), with a piezoelectric material who convert such mechanical energy into electricity (bio-inorganic generator). The mechanical stimuli, as pressure and vibration, are generated as an outcome of the produced gases coming from renewable fuels; this reaction is catalyzed by the biological machinery (enzymes) which are part of the generator. These devices are able to produce electricity at the microscopic scale starting from renewable fuels as sugars and alcohols coming from the biomass. This fact is very significant allowing the indirect production of electricity with zero emissions. Moreover, these bio-inorganic generators open a passionate spectrum of new applications in different fields like sensing, robotics and energetics.
\end{abstract}

Keywords: enzymes; bio-catalysis; energy; piezoelectricity; renewable fuels.

Citation: Velasco Lozano, S. y López Gallego, F. Bio-inorganic electricity generators. Conversion of renewable chemical energy driven by enzyme-functionalized piezoelectric materials. Tecnologia, Ciencia y Educación, 15, 125-139. 


\section{Sumario}

1. Introducción

1.1. Generadores y nanogeneradores

1.2. Biomáquinas como transformadores energéticos ideales

1.3. Combustibles renovables para alimentar generadores bioinorgánicos

2. Materiales y métodos

2.1. Materiales

2.2. Métodos

3. Resultados

3.1. Evaluación de la producción de gas mediante sistemas enzimáticos

3.2. Desempeño de los generadores bioinorgánicos

3.3. Producción de electricidad a partir de diferentes combustibles renovables a través de generadores bioinorgánicos a base de sistemas multienzimáticos

4. Conclusiones

Referencias bibliográficas

Nota: este trabajo se enmarca dentro de la Convocatoria de Ayudas a Proyectos de I+D+i 2017 de la Fundación Hergar (categoría: Investigación aplicada y tecnológica en ingenierías). 


\section{Introducción}

\subsection{Generadores y nanogeneradores}

La amenaza del calentamiento global y las crisis energéticas han motivado a la comunidad científica para buscar sistemas autosuficientes y limpios que permitan atender las necesidades energéticas de la sociedad actual. El diseño de nuevos sistemas de generación de energía a partir de fuentes renovables es una necesidad urgente para lograr un desarrollo sostenible (Krozer, 2013). A escala macroscópica, podemos disfrutar de sistemas competitivos de generación de electricidad a partir de la energía solar, geotérmica, eólica o hidráulica. Sin embargo, a escala microscópica, los sistemas de generación de energía están todavía en desarrollo. Posiblemente, las pilas de combustibles basadas en procesos electroquímicos son los sistemas más avanzados (Kirubakaran, Jain y Nema, 2009) y ya se visualizan algunas aplicaciones en un futuro cercano.

La revolución nanotecnológica ha potenciado la creación de sistemas capaces de producir energía a partir de diversas fuentes. Un claro ejemplo son los generadores de electricidad basados en nanomateriales. La mayoría de los nanogeneradores están basados en la producción de energía eléctrica como respuesta a diferentes estímulos mecánicos (diferencia de presión, temperatura o fricción) (Zi y Wang, 2017). Estos sistemas, igual que las nanopilas de combustible, se están convirtiendo en las fuentes de alimentación de los dispositivos del futuro, sobre todo para aquellos con aplicaciones en sensórica, nanorrobótica, sistemas microelectromecánicos, monitorización ambiental de forma remota e incluso en dispositivos electrónicos portátiles.

La mayoría de los generadores y nanogeneradores capaces de producir electricidad están basados en el efecto piezoeléctrico y en el efecto triboeléctrico. Los primeros están formados por materiales cristalinos sin centro de simetría que, al ser sometidos a fuerzas mecánicas (presión o cizallamiento), generan cargas eléctricas en su superficie. Estas cargas eléctricas se pueden aprovechar para generar una diferencia de potencial (Wang, 2012). Por el contrario, el efecto triboeléctrico consiste en la producción de cargas eléctricas como consecuencia del frotamiento de dos materiales con cargas opuestas (Fan, Tian y Lin Wang, 
2012). Desde el punto de vista de los nanomateriales, los nanogeneradores piezoeléctricos son mucho más interesantes, puesto que la composición y configuración de la nanoestructura piezoeléctrica va a definir la eficiencia del nanogenerador.

Los nanogeneradores piezoeléctricos pueden estar formados por diferentes tipos de materiales cristalinos inorgánicos, como $\mathrm{ZnO}, \mathrm{CdS}, \mathrm{GaN}_{0} \circ \mathrm{BaTiO}_{3}$. El primer nanogerador descrito data del año 2006, cuando el profesor Wang, en el Georgia Institute of Technology (EE. UU.), desarrolló nanohilos de óxido de zinc tipo $p$ ( $\mathrm{ZnO} \mathrm{NW}$, del inglés ZnO nanowires) capaces de producir electricidad tras ser mecánicamente deformados (Wang y Song, 2006).

Sin embargo, la mayoría de estos dispositivos requieren de un modo u otro la acción humana, lo que limita su aplicación autónoma. Por ejemplo, el grupo del profesor Wang ha desarrollado dispositivos flexibles basados en ZnO NW que son capaces de producir electricidad al doblarse o al ejercer presión sobre ellos. Estos dispositivos tienen un enorme interés en el desarrollo de paneles táctiles para dispositivos electrónicos o para la monitorización ambiental de parámetros físico-químicos o biológicos (Fu et al., 2017). Por el contrario, este tipo de dispositivos resultarían ineficientes

Este trabajo ha conseguido fundir los conceptos de «generador» y «pila de combustible» en un único dispositivo que sea capaz de transformar energía química en energía eléctrica a través de energía mecánica. Esto ha sido posible gracias al acoplamiento de un transformador de energía química en energía mecánica (enzimas) con un generador piezoeléctrico comercial a la hora de producir electricidad de manera continua, puesto que, o bien dependen del factor humano para generar una energía mecánica voluntaria, o bien del factor ambiental como fuente de energía mecánica no controlada y discontinua (véase figura 1). El escenario ideal sería un generador o nanogenerador que dependiese exclusivamente de una fuente de energía mecánica autónoma, controlada y continua. Este sería un escenario similar al que encontramos en las pilas de combustible, donde el combustible actúa como fuente de energía química que es transformada en energía eléctrica de forma autónoma, controlada y continua.

Este trabajo ha conseguido fundir los conceptos de «generador» y «pila de combustible» en un único dispositivo que sea capaz de transformar energía química en energía eléctrica a través de energía mecánica (véase figura 1). Esto ha sido posible gracias al acoplamiento de un transformador de energía química en energía mecánica (enzimas) con un generador piezoeléctrico comercial. Idealmente, este transformador debe ser altamente selectivo y limpio para lograr una elevada eficiencia de transformación energética sin generar residuos tóxicos para el medioambiente. La naturaleza es una fuente de inspiración a la hora de encontrar una maquinaria biomolecular que pueda actuar como un transformador energético ideal. Esta maquinaria debe ser capaz de convertir un combustible químico renovable en estímulos mecánicos (por ejemplo, presión o vibración mecánica) que sean capaces de doblar o comprimir el material piezoeléctrico que forma parte del nanogenerador, produciendo corriente 
eléctrica. Aunque hay ejemplos del uso de elementos biológicos en pilas de combustible (Luckarift, Atanassov y Johnson, 2014), este trabajo ha generado el primer precedente de acoplamiento directo de maquinarias moleculares biológicas a generadores piezoeléctricos.

Figura 1. Generadores bioinorgánicos como alternativa a los generadores inorgánicos actuales

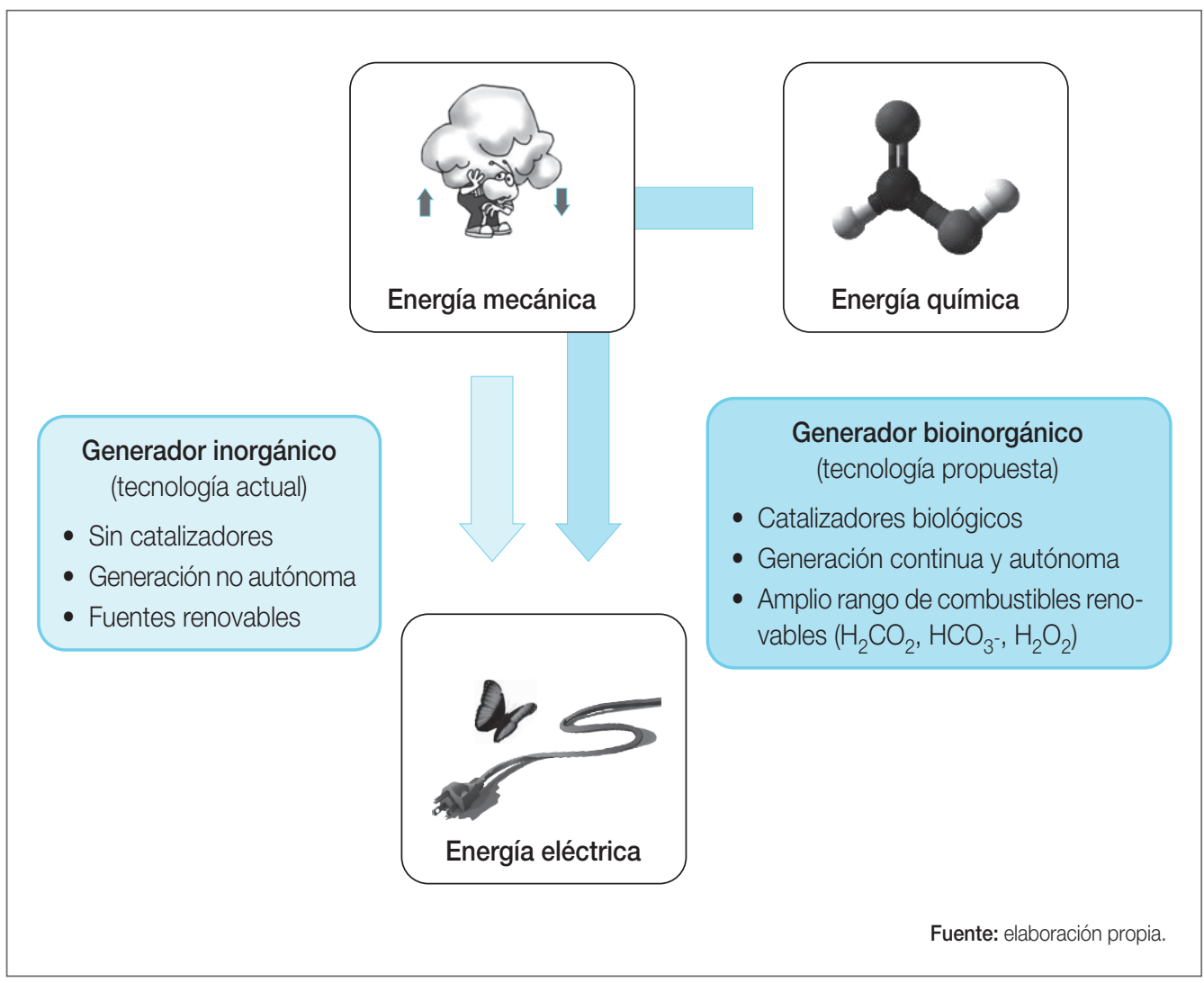

\subsection{Biomáquinas como transformadores energéticos ideales}

La naturaleza presenta numerosas maquinarias moleculares que actúan como transformadores energéticos ideales. Estos transformadores energéticos suelen tener un módulo catalítico y un módulo mecánico o estructural. El módulo catalítico suele ser un dominio enzimático
La naturaleza presenta numerosas maquinarias moleculares que actúan como transformadores energéticos ideales 
que transforma el combustible para generar la energía suficiente que permita un cambio conformacional en el módulo mecánico, dando lugar al movimiento o a algún otro efecto físico-químico. Estos sistemas biológicos han inspirado el diseño de sistemas exclusivamente químicos, capaces de transformar la energía química en energía mecánica, principalmente en movimiento. A estos sistemas se les denomina «nanomotores» moleculares y tienen gran aplicación en diversos campos como la medicina, la sensórica o la biorremediación (Rayment et al., 1993; Reinišová, Hermanová y Pumera, 2019; Zarei y Zarei, 2018). El gran potencial biotecnológico de los nanomotores ha despertado el interés de gran número de investigadores.

\section{El tipo de enzima propulsora más empleado hasta ahora es la catalasa, capaz de catalizar la transformación de peróxido de hidrógeno en oxígeno molecular formando burbujas}

Además, en los últimos años se han descrito los primeros sistemas híbridos quimioenzimáticos capaces de propulsar nanoestructuras. El tipo de enzima propulsora más empleado hasta ahora es la catalasa, capaz de catalizar la transformación de peróxido de hidrógeno en oxígeno molecular formando burbujas (Ma y Sánchez, 2017; Zhao, Norris y Liu, 2014). Asimismo, existen otras enzimas, como la ureasa, la acetilcolinesterasa, la glucosa oxidasa y la aldolasa, que han sido menos empleadas para propulsar nanopartículas (Arqué et al., 2019). Las burbujas de los gases producidos en presencia de surfactantes son las causantes de la propulsión del nanomaterial o del aumento de la presión de un fluido. Sin embargo, la parte biológica de estos sistemas ha quedado restringida a la catalasa como maquinaria propulsora, al peróxido de hidrógeno como combustible y al $\mathrm{O}_{2}$ como gas propulsor.

Gracias a la diversidad de actividades enzimáticas y a la versatilidad de las herramientas para su mejora, es plausible pensar en nuevos transformadores biomoleculares que son capaces de usar combustibles más sostenibles que el peróxido de hidrógeno. Hay numerosos tipos de enzimas capaces de generar incrementos de presión o incluso movimiento mediante la liberación controlada de gases $\left(\mathrm{O}_{2}, \mathrm{CO}_{2}, \mathrm{~N}_{2} \circ \mathrm{H}_{2}\right)$ a partir de la transformación química de combustibles. Enzimas del tipo descarboxilasas, nitrogenasas e hidrogenasas complementarían a las catalasas como maquinarias moleculares para producir energía mecánica a partir del burbujeo de diferentes gases.

\subsection{Combustibles renovables para alimentar generadores bio- inorgánicos}

Para generar energía mecánica, que después será convertida en energía eléctrica por un generador bioinorgánico, serán necesarios combustibles renovables y económicamente com- 
petitivos. Estos combustibles serán transformados en energía mecánica por un biotransformador molecular, generando un gas que ejercerá una fuerza mecánica, bien sea una presión o una vibración, sobre un generador inorgánico que transformará esa energía mecánica en energía eléctrica. La electricidad producida se podrá usar para alimentar diferentes dispositivos.

El peróxido de hidrógeno es el combustible utilizado por la mayoría de los dispositivos químicos que son capaces de mover nanoestructuras mediante propulsión con $\mathrm{O}_{2}$. Sin embargo, su síntesis química conlleva la generación de grandes cantidades de residuos (Gao y Wang, 2014). Esto hace que el peróxido de hidrógeno, debido a su proceso de síntesis, no sea un combustible medioambientalmente sostenible para la producción de energía eléctrica. Como alternativa a la síntesis química, se puede producir peróxido de hidrógeno a partir de glucosa mediante un único paso catalizado por una enzima: la glucosa oxidasa, que es capaz de oxidar la glucosa produciendo ácido glucónico y peróxido de hidrógeno (Orozco et al. , 2013). Además, nuestro grupo de investigación ha descrito recientemente un sistema de producción de peróxido de hidrógeno a partir de ácido fórmico (Campos-Martín, BlancoBrieva y Fierro, 2006). De este modo, la síntesis enzimática del peróxido de hidrógeno a partir de fuentes renovables convertiría esta molécula en un combustible sostenible para lograr una producción limpia de electricidad.

En este trabajo, por tanto, hemos desarrollado dispositivos bioinorgánicos capaces de generar electricidad a partir de diferentes fuentes renovables de energía química mediante el diseño y la creación de sistemas multienzimáticos que catalizan la transformación de compuestos renovables en gases capaces de generar energía mecánica. Después, estos sistemas multienzimáticos se han acoplado a generadores bioinorgánicos que son capaces de producir electricidad a partir de estímulos mecánicos (burbujas) provenientes de combustibles renovables. Este diseño se presenta como una alternativa a las biopilas de combustible, donde materias primas electroquímicamente activas son capaces de generar una corriente eléctrica.

\author{
En este trabajo hemos desarrollado \\ dispositivos bioinorgánicos capaces \\ de generar electricidad a partir de \\ diferentes fuentes renovables de \\ energía química mediante el diseño y la \\ creación de sistemas multienzimáticos \\ que catalizan la transformación de \\ compuestos renovables en gases \\ capaces de generar energía mecánica
}

\section{Materiales y métodos}

\subsection{Materiales}

Reactivos y sustratos como el peróxido de hidrógeno, la glucosa, el ácido 2,2'-azinobis(3-etilbenzotiazolino-6-sulfónico [ABTS]), la D-alanina y la L-fenilalanina fueron adquiridos 
de Sigma-Aldrich (San Luis, Misuri, EE. UU.). Las enzimas -catalasa (CAT) de hígado bovino, glucosa oxidasa (GOX) de Aspergillus niger, alcohol oxidasa (AOX) de Hansenula sp., D-aminoácido oxidasa (DAO) de Trigonopsis variabilis, L-aminoácido oxidasa (LAO) de Crotalus adamanteus tipo I y peroxidasa de rábano (HRP)- también fueron adquiridas de SigmaAldrich. Los discos piezoeléctricos fabricados con zirconato titanato de plomo (lead zirconate titanate [PZT] $)(6,3 \mathrm{kHz}, 1 \mathrm{~K} \Omega, 0,01 \mu \mathrm{F}, 20 \mathrm{~mm} \times 0,42 \mathrm{~mm}$ [cat. 7BB-20-6I0, Murata]) fueron adquiridos de Farnell Element14 Components (Barcelona, España).

\subsection{Métodos}

Producimos y purificamos seis diferentes preparaciones enzimáticas, las cuales fueron sobreexpresadas en células Escherichia coli y purificadas mediante resinas activadas con cobalto. El material piezoeléctrico empleado para la fabricación de los generadores bioinorgánicos descritos en este proyecto fue un disco comercial compuesto por una capa de PTZ con un electrodo inferior de cobre y un electrodo superior de plata. Adaptamos dicho disco PZT a dos tipos de dispositivos: a un generador bioinorgánico cerrado (CBIG) y a un generador bioinorgánico abierto (OBIG), integrando el mismo disco PZT al fondo de una columna cilíndrica de plástico, permaneciendo abierta al medioambiente por la parte superior.

En la arquitectura de CBIG (véase figura 2 a), la mezcla de reacción nunca entra en contacto directo con el material piezoeléctrico colocado en la parte superior de la cámara sellada. Una vez que añadimos el sustrato combustible (min 1), la enzima CAT oxida el combustible $\left(\mathrm{H}_{2} \mathrm{O}_{2}\right)$, generando oxígeno molecular que incrementa la presión de gas del espacio de cabeza entre el nivel del líquido (medio de reacción) y la superficie del disco PZT (véase figura 2 b), zona negra). Después de varios minutos de operación, se despresuriza la cámara por apertura de una válvula manual que libera los gases acumulados en el espacio de cabeza durante la reacción enzimática (véase figura 2 b), zona azul). Para cuantificar la respuesta de salida eléctrica obtenida de ambas formas (presurización y despresurización), llevamos a cabo el registro del voltaje de circuito abierto a lo largo del tiempo de operación calculando la energía eléctrica generada de acuerdo a la ecuación 1 (Briscoe et al., 2013).

\section{Ecuación 1. Energía eléctrica generada}

$$
E=\int_{t_{1}}^{t_{2}} \frac{V^{2}(t)}{R} d t
$$

Donde $E$ es la energía eléctrica generada, $V$ es el voltaje generado desde el inicio $\left(t_{1}\right)$ al final $\left(t_{2}\right)$ de un ciclo de operación, empleando una resistencia constante $(R)$. Fijamos el valor de $R$ en $60 \mathrm{M} \Omega$ (medidos experimentalmente en condiciones de máximo voltaje producido). De modo interesante, hacemos notar que se obtiene casi la misma energía eléctrica al pre- 
surizar (véase figura 2 b), zona negra) que al despresurizar (véase figura 2 b), zona azul) CBIG (0,18 y $0,20 \mathrm{~nJ} \times \mathrm{cm}^{2}$, respectivamente). Sin embargo, los máximos voltajes alcanzados para dichos procesos son distintos.

En el caso del OBIG, el disco PZT se encuentra en contacto directo con la mezcla acuosa de reacción que es colocada al fondo de la columna (véase figura 2 c). Este sistema abierto permite recolectar la energía mecánica generada por el borboteo de los gases producidos por las enzimas en vez de responder a incrementos de presión. El estímulo mecánico del borboteo de los gases es traducido como una vibración que resulta en una salida de voltaje a lo largo de la reacción enzimática (véase figura 2 d), zona azul).

Figura 2. Arquitectura de los generadores bioinorgánicos

a)

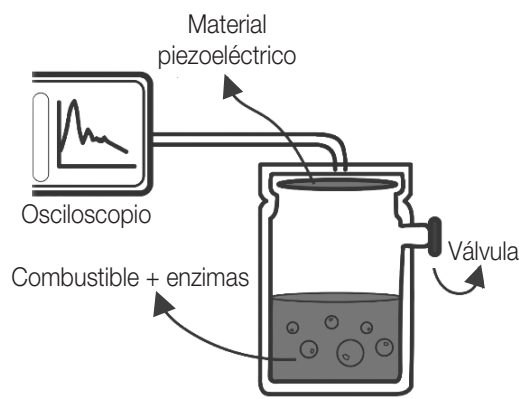

c)

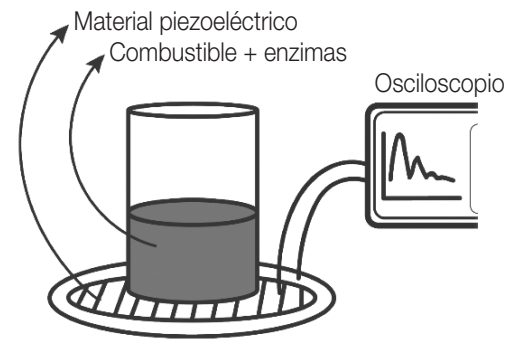

b)

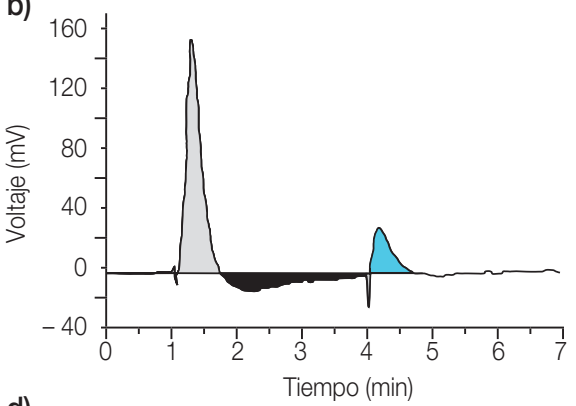

d)

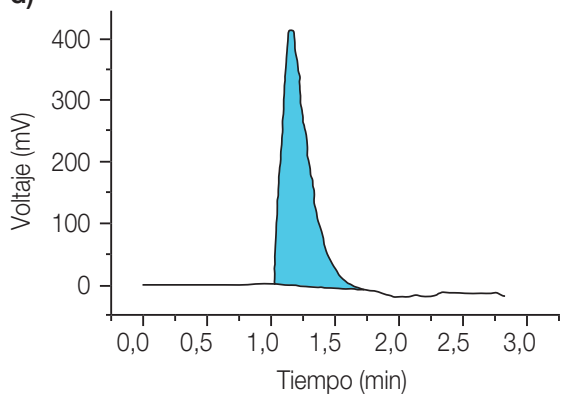

Nota.

a) CBIG, vista en corte transversal.

b) Respuesta de salida eléctrica de CBIG. Temporización: 0-1 min (estabilización del sistema), 1-1,1 min (administración del sustrato [gris claro]), 1,8 min (cerrado de válvula), 1,8-4 min (producción de gas que genera efecto de presurización [negro]), 4 min (apertura de la válvula) y 4-5 min (liberación del gas, efecto de despresurización [azul]).

c) OBIG

d) Respuesta de salida eléctrica de OBIG. Temporización: 0-1 min (estabilización del sistema), 1 min (adición del sustrato) y 1-1,7 min (efecto vibracional desencadenado por el borboteo [azul]). 


\section{Resultados}

\subsection{Evaluación de la producción de gas mediante sistemas enzi- máticos}

Se han probado 12 sistemas enzimáticos para la producción de gas a partir de diferentes materias primas renovables. El sistema enzimático de mayor y más veloz producción de gas fue el compuesto por la CAT, desplazando un volumen de 1,5 $\mathrm{mL}$ de $\mathrm{O}_{2}$ en $5 \mathrm{~min}$. Todos los demás sistemas produjeron alrededor de 20 veces menos volumen de gas. Por otro lado, los sistemas bienzimáticos compuestos por una oxidasa y catalasa también fueron capaces de producir gas y generar borboteo de la solución acuosa cuando fueron suplementados con pequeñas cantidades de $\mathrm{H}_{2} \mathrm{O}_{2}$.

\subsection{Desempeño de los generadores bioinorgánicos}

Evaluamos la generación de energía mediante los sistemas CBIG y OBIG, observando que el sistema abierto generaba una mayor cantidad de energía y tenía un modo de trabajo mucho más simplificado. Por esto decidimos continuar el desarrollo con el sistema OBIG.

En este sistema abierto, la producción de energía eléctrica depende tanto de la concentración de sustrato como del volumen o cantidad de sustrato adicionados (véase figura 3). OBIG produce mayor energía eléctrica al aumentar la concentración de enzima (véase figura 3 a), donde la máxima producción se logra de 400-600 mM de combustible $\mathrm{H}_{2} \mathrm{O}_{2}$ y $88 \mu \mathrm{g} \mathrm{x} \mathrm{mL} \mathrm{m}^{-1} \mathrm{CAT}$ (véase figura $3 \mathrm{~b}$ ). Esta producción de energía se traduce en una salida de voltaje máximo de $918 \mathrm{mV}$, generando $265 \mathrm{~nJ} \times \mathrm{cm}^{-2}$.

El sistema OBIG puede operar con diferentes volúmenes de combustible; sin embargo, el sistema es menos productivo conforme aumenta el volumen de trabajo, por lo que se obtiene la misma energía eléctrica a volúmenes mayores de $1 \mathrm{~mL}$. Este estudio demuestra que, para una mayor producción de energía eléctrica, la mayor concentración de combustible es más efectiva que la mayor cantidad total de combustible.

En las mejores condiciones, OBIG opera óptimamente con $200 \mu \mathrm{L}$ de volumen de com-

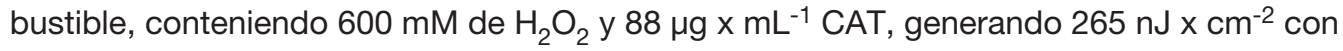
una salida máxima de voltaje de circuito abierto de $0,9 \mathrm{~V}$ y una productividad de $1,11 \mu \mathrm{J} x$ $x \mathrm{mmol}^{-1} \mathrm{x} \mathrm{cm}^{-2}$. Esta respuesta de salida eléctrica puede traducirse a $4,4 \mathrm{nW} \mathrm{x} \mathrm{cm}{ }^{-2}$, cuya magnitud está dentro de las densidades de energía requeridas para alimentar varios nanosensores reportados hoy en día (83 nW x cm-2) (Wang et al., 2005). Además, OBIG puede producir energía eléctrica después de 20 ciclos de adición de combustible con la misma enzima, disminuyendo hasta un $30 \%$ de la inicial. 
Figura 3. Efecto de la concentración de CAT (a) y del sustrato (b) en la energía producida (cuadros negros) y el voltaje producido (cuadros blancos) usando OBIG

a)

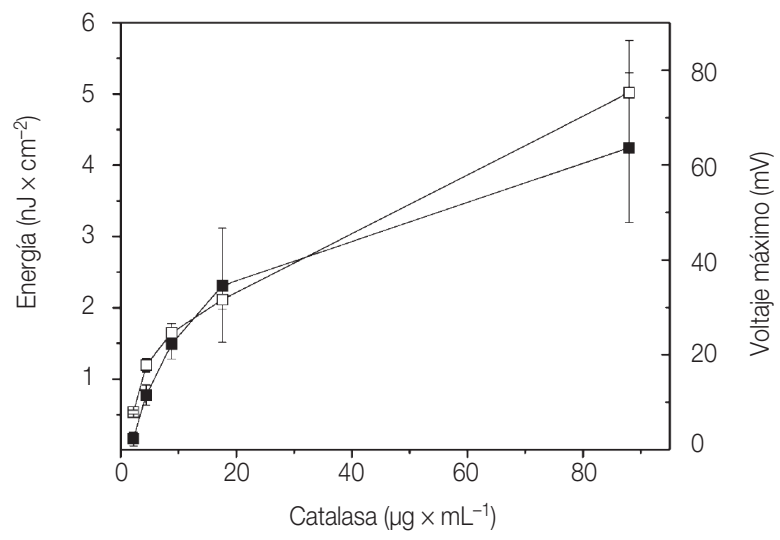

b)

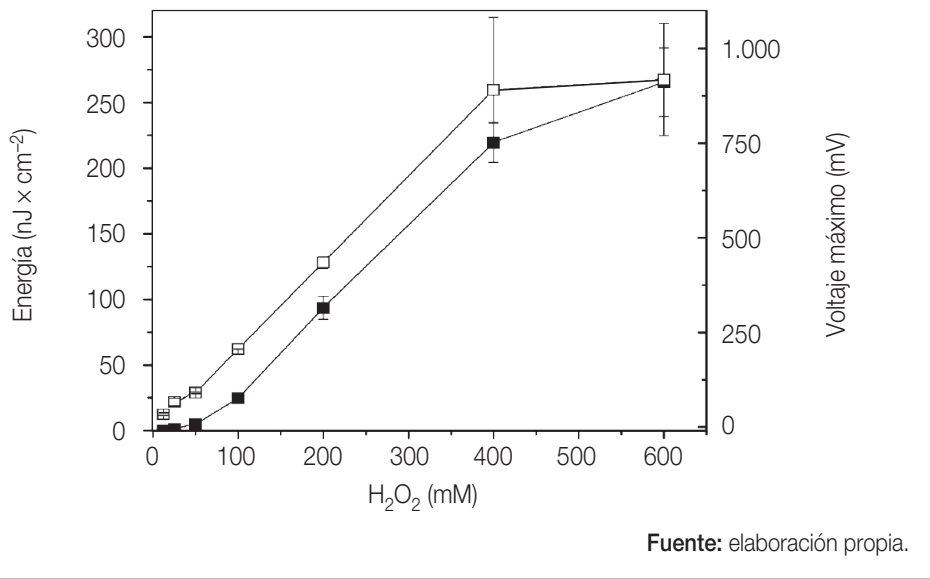

\subsection{Producción de electricidad a partir de diferentes combus- tibles renovables a través de generadores bioinorgánicos a base de sistemas multienzimáticos}

Con el objetivo de expandir este concepto a otros combustibles renovables, se probó la generación de energía mecánica (burbujas) con otros sistemas enzimáticos. Los sistemas bienzimáticos estudiados fueron los compuestos por una oxidasa y CAT. Con dicho sistema bienzimático es posible generar in situ el $\mathrm{H}_{2} \mathrm{O}_{2}$ a partir de una gran diversidad de sustratos oxidables, como azúcares, aminoácidos y alcoholes, etc. El peróxido de hidrógeno producido in situ es convertido en agua y burbujas de oxígeno mediante la acción de la CAT, generando así vibración. 
Para demostrar este concepto, en un principio estudiamos el sistema glucosa oxidasa (GOX) y CAT. En dicho sistema, la enzima GOX oxida la glucosa produciendo gluconato y $\mathrm{H}_{2} \mathrm{O}_{2}$, siendo este último convertido en agua y oxígeno por la CAT.

Al comparar el desempeño de OBIG acoplado al sistema CAT o al sistema GOX/CAT, el sistema bienzimático genera seis veces mayor energía eléctrica a partir de glucosa $100 \mathrm{mM}$ que cuando solo se emplea CAT y la misma concentración inicial de $\mathrm{H}_{2} \mathrm{O}_{2}$. Del mismo modo, el máximo voltaje alcanzado fue dos veces mayor con el sistema bienzimático. La productividad fue del doble. Estudiamos las condiciones óptimas de producción de energía eléctrica operando OBIG con el sistema bienzimático GOX/CAT, alcanzando hasta $26 \mathrm{~nJ} \mathrm{x} \mathrm{\textrm {cm } ^ { - 2 }}$ y un voltaje de $0,159 \mathrm{~V}$ con una máxima productividad de $443 \mathrm{~nJ} \mathrm{x} \mathrm{mmol}{ }^{-1} \mathrm{x} \mathrm{cm}^{-2}$. Estos valores indican que el uso de glucosa como co-combustible mejora la producción de energía del sistema hasta seis veces en comparación con el sistema que no emplea glucosa.

Posteriormente, evaluamos la producción de energía eléctrica a partir de otras fuentes renovables, usando pares enzimáticos catalasa/oxidasa (véase figura 4). El sistema bienzimático que mayor producción eléctrica y productividad logró fue el compuesto por la DAO/ CAT. Atribuimos estos mejores resultados con este sistema a que la enzima DAO tiene menor constante de inhibición frente al $\mathrm{H}_{2} \mathrm{O}_{2}$ comparado con las otras oxidasas, además de que tiene la menor $\mathrm{K}_{\mathrm{M}}$ hacia su sustrato (co-combustible) en comparación con las demás oxidasas probadas en este estudio. Con este sistema bienzimático se puede producir energía eléctrica empleando aminoácidos como co-combustible.

Figura 4. Diferentes sistemas oxidasa/catalasa acoplados a OBIG

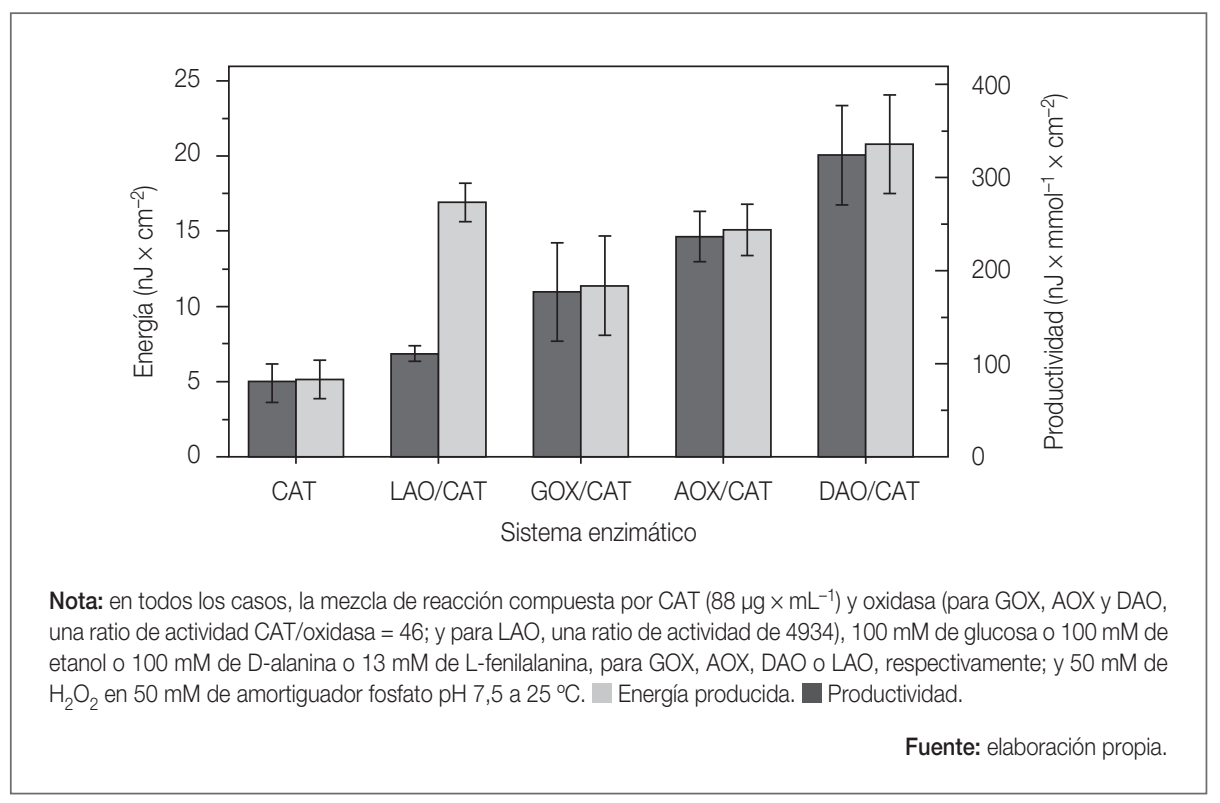




\section{Conclusiones}

En este trabajo hemos diseñado y fabricado unos nuevos generadores bioinorgánicos que son capaces de producir electricidad a partir de combustibles químicos renovables. Estos sistemas están basados en enzimas capaces de convertir energía química de algunas moléculas (por ejemplo, peróxido de hidrógeno) en energía mecánica que es simultáneamente transformada en energía eléctrica mediante la acción de un material piezoeléctrico. La arquitectura desarrollada en este trabajo permite usar diferentes combustibles renovables mediante la selección adecuada del sistema enzimático que se debe acoplar al material piezoeléctrico. Gracias al acoplamiento de oxidasas dependientes de flavinas con la enzima catalasa, somos capaces de aumentar la eficiencia y maximizar la generación de energía a partir de un recurso tan renovable como la glucosa. Esta tecnología tan prometedora abre una batería de oportunidades para la aplicación de materiales piezoeléctricos y enzimas en el campo de la generación de electricidad. Además, la expansión de este concepto a sistemas enzimáticos más complejos conducirá a la obtención de otro tipo de gases que permitan estímulos mecánicos más vigorosos que den lugar a valores de producción energética más altos, así como materiales piezoeléctricos que faciliten minimizar las pérdidas de conversión energética en el proceso. En este sentido, nosotros vislumbramos que el futuro de esta tecnología debería encaminarse hacia su implementación en sistemas fluodinámicos que permitiesen una producción de energía continua, para tener un control más preciso de la demanda y así poderla adaptar a diferentes dispositivos con suministro de energía autónomo a partir de un reservorio de combustible.

\section{Referencias bibliográficas}

Arqué, X., Romero-Rivera, A., Feixas, F., Patiño, T., Osuna, S. y Sánchez, S. (2019). Intrinsic enzymatic properties modulate the self-propulsion of micromotors. Nature Communications, 10(1).

Briscoe, J., Jalali, N., Woolliams, P., Stewart, M., Weaver, P. M., Cain, M. y Dunn, S. (2013). Measurement techniques for piezoelectric nanogenerators. Energy \& Environmental Science, 6, 3.035-3.045.

Campos-Martín, J. M., Blanco-Brieva, G. y Fierro, J. L. G. (2006). Hydrogen peroxide synthesis: an outlook beyond the anthraquinone process. Angewandte Chemie International Edition, 45, 6.962-6.984.

Fan, F.-R., Tian, Z.-Q. y Lin Wang, Z. (2012). Flexible triboelectric generator. Nano Energy, 1(2), 328-334.

Fu, Y. Q., Luo, J. K., Nguyen, N. T., Walton, A. J., Flewitt, A. J., Zu, X. T., ... y Milne, W. I.
(2017). Advances in piezoelectric thin films for acoustic biosensors, acoustofluidics and lab-on-chip applications. Progress in Materials Science, 89, 31-91.

Gao, W. y Wang, J. (2014). The environmental impact of micro/nanomachines: a review. ACS Nano, 8(4), 3.170-3.180.

Kirubakaran, A., Jain, S. y Nema, R. K. (2009). A review on fuel cell technologies and power electronic interface. Renewable and Sustainable Energy Reviews, 13(9), 2.430-2.440.

Krozer, Y. (2013). Cost and benefit of renewable energy in the European Union. Renewable Energy, 50, 68-73.

Luckarift, H. R., Atanassov, P. B. y Johnson, G. R. (2014). Enzymatic uel Cells: From Fundamentals to Applications. Wiley.

Ma, X. y Sánchez, S. (2017). Bio-catalytic mesoporous Janus nano-motors powered 
by catalase enzyme. Tetrahedron, 73(33), 4.883-4.886.

Orozco, J., García-Gradilla, V., D’Agostino, M., Gao, W., Cortés, A. y Wang, J. (2013). Artificial enzyme-powered microfish for waterquality testing. ACS Nano, 7(1), 818-824.

Rayment, I., Holden, H. M., Whittaker, M., Yohn, C. B., Lorenz, M., Holmes, K. C. y Milligan, R. A. (1993). Structure of the actin-myosin complex and its implications for muscle contraction. Science, 261(5.117), 58-65.

Reinišová, L., Hermanová, S. y Pumera, M. (2019). Micro/nanomachines: what is needed for them to become a real force in cancer therapy? Nanoscale, 11, 6.519-6.532.

Wang, X. (2012). Piezoelectric nanogeneratorsÇharvesting ambient mechanical energy at the nanometer scale. Nano Energy, 1(1), 13-24.
Wang, Z. L. y Song, J. (2006). Piezoelectric nanogenerators based on zinc oxide nanowire arrays. Science, 312(5.771), 242-246.

Wang, X., Song, J., Li, P., Ryou, J. H., Dupuis, R. D., Summers, C. J. y Wang, Z. L. (2005). Growth of uniformly aligned $\mathrm{ZnO}$ nanowire heterojunction arrays on GaN, AIN, and $\mathrm{Al}_{0.5} \mathrm{Ga}_{0.5} \mathrm{~N}$ substrates. Journal of the American Chemical Society, 127(21), 7920-7923.

Zarei, M. y Zarei, M. (2018). Self-propelled micro/nanomotors for sensing and environmental remediation. Small, 14.

Zhao, X., Norris, S. J. y Liu, J. (2014). Molecular architecture of the bacterial flagellar motor in cells. Biochemistry, 53(27), 4.323-4.333.

Zi, Y. y Wang, Z. L. (2017). Nanogenerators: an emerging technology towards nanoenergy. APL Materials, 5(7).

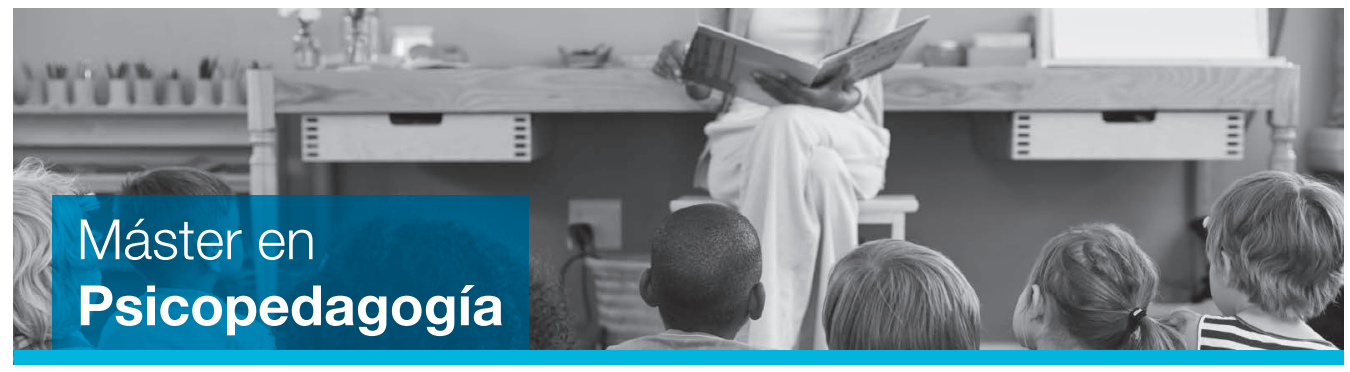

Este máster oficial [60 créditos ECTS] tiene una duración normal de 12 meses.

Dirigido a: Personas vinculadas con el mundo de la educación formal y no formal que deseen actualizar sus conocimientos. El estudiante de este máster ha de estar interesado por la labor del educador en un enfoque educativo inclusivo en el ámbito de la educación formal, y en el trabajo con diferentes grupos o colectivos sociales, favoreciendo la mejora de sus condiciones de vida y la disminución de las desigualdades por motivos de carácter social y cultural.

Objetivos: Permite el desempeño de una labor profesional especializada, avanzada y focalizada en el análisis, la planificación y la intervención para la mejora de los contextos educativos, sociolaborales y sociocomunitarios, de ahí la necesidad de una formación de posgrado que permita el desarrollo de las competencias específicas y multidisciplinares requeridas para su práctica profesional. Pretende dar cobertura a las funciones básicas de los psicopedagogos en distintos ámbitos. 
Para los Grados en Derecho o en

Ciencias del Trabajo, Relaciones

Laborales y Recursos Humanos

- Administración y Finanzas

- Asistencia a la Dirección

- Secretariado

\section{Para los Grados en Administración y}

Dirección de Empresas o en Economía

- Administración y Finanzas

- Asistencia a la Dirección

- Comercio Internacional

- Gestión Comercial y Marketing

- Marketing y Publicidad

- Secretariado

- Transporte y Logística

\section{Para el Grado en Marketing}

- Administración y Finanzas

- Comercio Internacional

- Gestión Comercial y Marketing
- Gestión de Alojamientos Turísticos

- Gestión de Ventas y Espacios Comerciales

- Marketing y Publicidad

- Transporte y Logística

Para los Grados en Magisterio de Educación Infantil y Magisterio de Educación Primaria

- Animación de Actividades Físicas y Deportivas

- Educación Infantil

- Integración Social

- Animación Sociocultural y Turística

Para el Grado en Empresas y Actividades Turísticas

- Gestión Comercial y Marketing

- Gestión de Ventas y Espacios Comerciales

- Gestión de Alojamientos Turísticos

- Agencias de Viajes y Gestión de Eventos

- Guía, Información y Asistencias Turísticas

\footnotetext{
* De acuerdo a lo establecido en el Real Decreto 1618/2011, de 14 de noviembre, sobre reconocimiento de estudios en el ámbito de la educación superior.
} 


\section{Ingeniería de Tecnologías y Servicios de Telecomunicación}

El papel estratégico del sector de las TIC, y la aplicación creciente de estas en los distintos sectores de la sociedad, ha hecho aumentar la necesidad de profesionales de la telecomunicación, una demanda que crecerá exponencialmente en los próximos años. Este grado habilita para el ejercicio de la profesión de ingeniero técnico de telecomunicación, otorgando las competencias necesarias para conseguir las atribuciones profesionales de un ingeniero técnico de telecomunicación y ofreciendo una formación que capacita al estudiante a la hora de analizar, diseñar, implementar, explotar y gestionar sistemas, componentes y procesos del ámbito de las TIC.

\section{Psicología (Rama CC. de la Salud)}

Siguiendo el modelo científico-profesional de psicólogo (o scientist-practitioner), se trata de aportar a los alumnos los conocimientos científicos necesarios para comprender, interpretar, analizar y explicar el comportamiento humano, así como para evaluar e intervenir en el ámbito individual y social, con el fin de que los psicólogos y la psicología promuevan y mejoren la salud y la calidad de vida de las personas.

\section{Historia}

Se conjugan los conocimientos humanísticos básicos y generalistas con el aprendizaje de las herramientas y técnicas de las nuevas TIC. Los estudiantes adquirirán la formación, los conocimientos y las habilidades necesarias para permitirles el pleno desarrollo de las funciones relacionadas con la investigación y la enseñanza de la historia, con la finalidad de que comprendan y hagan comprensibles a los demás los acontecimientos del pasado.

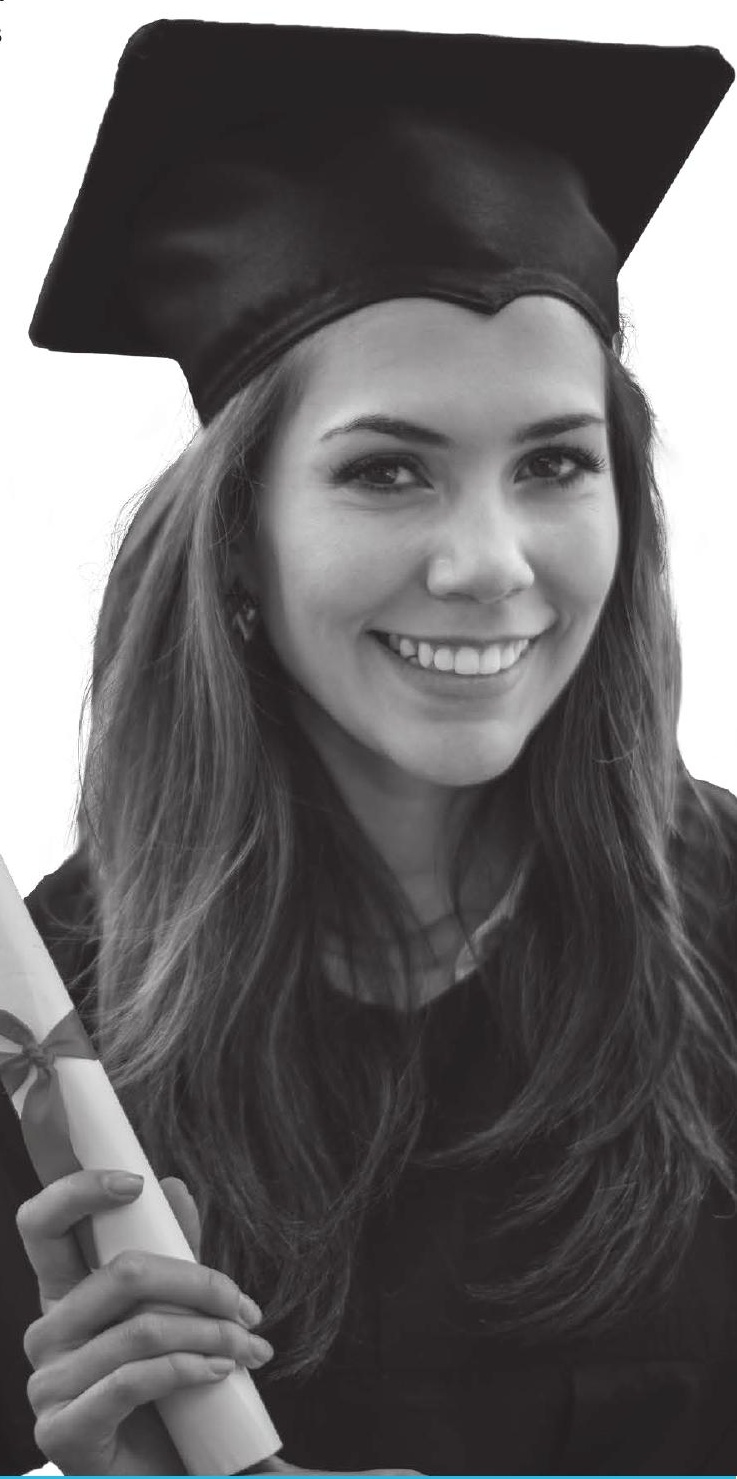

